\title{
Nostalgias barriales: Diferencias generacionales en tres barrios de Valparaíso
} [Neighborhood nostalgia: Generational differences in three neighborhoods in Valparaíso]

\author{
Clément Colin 1 (1)
}

${ }^{1}$ Pontificia Universidad Católica de Valparaíso

\begin{abstract}
Resumen
El artículo discute resultados de una investigación sobre las experiencias nostálgicas del cambio socio-urbano vividas por personas de distintas generaciones perteneciendo a familias de larga estancia en tres barrios de Valparaíso. A partir de un trabajo de campo realizado en 2019 y 2020, analiza los recuerdos, los imaginarios y los anhelos que personas de distintas generaciones asocian con este sentimiento. Discute así la idea de "nostalgia estructural" propuesta por Herzfeld, según la cual existiría una nostalgia que podría ser entendida como un recuerdo social transmitido entre generaciones. En este marco, muestra que las personas entrevistadas dan un sentido distinto a este sentimiento según su edad y sus experiencias pasadas. En suma, el artículo evidencia como el estudio de las nostalgias hace aparecer espacios generacionales que se superponen y a veces se contradicen a la escala de un mismo barrio.
\end{abstract}

Palabras clave: nostalgia, espacio generacional, significado, espacio vivido, barrio.

\begin{abstract}
In this article we discuss results of research on the nostalgic experiences of urban change encountered by people of different generations of long-stay families from three neighborhoods in Valparaíso, Chile. Based on fieldwork conducted in 2019 and 2020, we analyze the memories, imaginaries, and longings that people of different generations associate with this feeling. We discuss the idea of "structural nostalgia" proposed by Herzfeld, according to which the experience of nostalgia could be understood as a social memory transmitted between generations. Within this framework, we find that the people interviewed ascribe a different meaning to this feeling according to their age and past experiences. In sum, the article shows how the study of nostalgia reveals generational spaces that overlap and sometimes contradict one another at the scale of a single neighborhood.
\end{abstract}

Keywords: nostalgia, generational space, meaning, lived space, neighborhood.

Contacto: La comunicación sobre este artículo debe ser enviada a Clément Colin, email clement.colin@pucv.cl

Financiamiento: Esta investigación contó con financiamiento del Fondo Nacional de Desarrollo Científico y Tecnológico (FONDECYT 11180372) de la Agencia Nacional de Investigación y Desarrollo (ANID).

Agradecimientos: El autor agradece a Alexandra Benitt, Macarena Rojas, Natalie Calderón y Karina Ureta por su colaboración en el desarrollo del terreno y del análisis de los relatos y discursos. 


\section{INTRODUCCIÓN}

En la actualidad, la nostalgia es omnipresente tanto en los discursos políticos como en las prácticas cotidianas. Mientras algunos autores explican este fenómeno por el contexto socioeconómico y político global cada vez más incierto e inseguro (Appadurai, 1996), otros lo califican de epidemia global de nostalgia (Bauman, 2017). En esta perspectiva, la nostalgia ha sido así muchas veces interpretada como una reacción conservadora a la modernidad y vinculada con un deseo de retorno al pasado. Sin embargo, algunos autores mostraron el carácter plural y ambiguo de este sentimiento que no significa solamente un deseo por reproducir un pasado, sino también un compromiso con el presente pues se vincula y se activa desde valores, representaciones e imaginarios que se desplieguen en la actualidad en relación con pasados recordados y reinterpretados (Boym, 2001; Davis, 1979; May, 2017; Pickering \& Keightley, 2006). Frente a una idea de nostalgia conservadora o restauradora, proponen definiciones complementarias y muy distintas de la nostalgia, como reinvención y reconstrucción de sí mismo en el presente y para el futuro en la base de una mirada crítica sobre el pasado y el tiempo (Boym, 2001). En esta perspectiva, algunos autores la abordan como una reconstrucción que no se hace solamente en el tiempo sino también en el espacio. La estudian como una emoción que se encarna en las materialidades y en prácticas que vinculan los individuos con su entorno social y físico (Blunt, 2003; Bonnett \& Alexander, 2013; Bonnett, 2015; Kitson \& McHugh, 2015). Este artículo sigue esta línea de investigación para abordar la nostalgia como entrada para estudiar las experiencias de los cambios socio-urbanos vividos por los habitantes de la ciudad contemporánea.

En Chile, en los últimos años, los cambios sociourbanos han conducido a la multiplicación y a la difusión de discursos nostálgicos sobre tiempos pasados mejores. Este sentimiento se basa en particular en el deseo de reencontrar una vida barrial perdida debido a la fragmentación socioterritorial, al individualismo, y a la desconfianza generados por la aplicación del modelo urbano neoliberal (Letelier, 2018; Tapia, 2018). En la literatura, se observa que la aplicación de este modelo en las políticas urbanas ha conducido a una desaparición progresiva del espacio público como lugar de encuentro y su transformación en frontera entre grupos de habitantes que no tienen ni la misma historia ni las mismas expectativas por el futuro. Es en este contexto que surge la nostalgia por el barrio como base emocional a una demanda social y política cada vez más importante para hacer barrio y comunidad (Colin, 2017).

Para profundizar en la comprensión de esta añoranza por la vida barrial de antes, este artículo se interesa en las relaciones nostálgicas que personas de distintas generaciones desarrollan con su barrio en Valparaíso, Chile. El trabajo de campo fue realizado en 2019 y 2020 con miembros de familias antiguas (entre 40 y 70 años en el mismo barrio) de tres sectores de Valparaíso: el cerro Esperanza, el cerro Barón y el sector Playa Ancha Alto. 
Los cerros Esperanza y Barón son dos barrios históricamente obreros que se desarrollaron a partir del siglo XIX. Hoy día, son sectores residenciales compuestos respectivamente de 7,972 y 7,630 habitantes (Censo 2017). El barrio Esperanza está habitado por una población de categoría socioeconómica media y media alta, y el barrio Barón por una población de categoría media y media baja. El tercer sector de estudio se ubica en la parte alta del cerro Playa Ancha que se urbaniza durante la segunda parte del siglo XX a partir de una planificación urbana conducida por el Estado chileno para luchar contra el problema de acceso a la vivienda. Es un territorio que agrupa 14,241 habitantes (Censo 2017) divididos en distintos sectores que fueron construidos progresivamente en diferentes periodos desde los años 1960 hasta los años 1980. En este sector, los habitantes pertenecen a las categorías socioeconómicas baja y media baja. Este trabajo participa del campo de los estudios emocionales, mostrando cómo aporta en la comprensión de realidades sociales actuales. Desarrollado primero desde la antropología y la sociología norteamericana (Hochschild, 1983; Kemper, 1978; Lutz \& White, 1986; Thoits, 1989), este campo tiene una fuerte resonancia en el mundo latinoamericano (Aguilar \& Soto, 2013; Ramírez \& Aguilar, 2006; Scribano \& Aranguren, 2017) y chileno (Campos et al., 2017; Pincheira, 2019). Siguiendo esta línea, el artículo pone en el centro de la discusión las ideas de emoción y de experiencia para abordar las formas en que personas viven, piensan y se piensan en relación con su entorno social-material cotidiano. Desde el estudio de las nostalgias, el artículo contribuye en una mejor comprensión de las tensiones generacionales existentes a escala barrial y cómo esas se materializan en los relatos y las experiencias de los sujetos, influyendo a su vez la forma de pensar y relacionarse en/con el barrio actual.

Compuesta etimológicamente por dos palabras griegas -nostos, el regreso, y algos, el sufrimientola nostalgia es un concepto creado en el siglo XVII por el suizo Johannes Hofer para caracterizar un fenómeno medicinal considerado como una patología psicológica (Davis, 1979). Al final del siglo XX, la nostalgia fue muchas veces entendida como una resistencia contra el cambio basada en una idealización del pasado (Shaw \& Chase, 1989). En otras palabras, ha sido principalmente abordada en su dimensión restauradora (Boym, 2001), como emoción relacionada con la idea de regreso y con las ansiedades, incertidumbres, y temores causados por un sentimiento de pérdida de identidad (Appadurai, 1996). Sin embargo, la nostalgia es emoción plural ambivalente porque se puede referir tanto a un deseo de retorno basado en recuerdos vividos y/o compartidos como a una proyección basada en una crítica del presente en relación con pasados desaparecidos (Colin et al., 2019). En esta perspectiva, como otros trabajos (Boym, 2001; Davis, 1979; Pickering \& Keightley, 2006), aquí se aborda este sentimiento no solamente como un deseo de regreso a un pasado perdido, sino también como intrínsecamente vinculado con los valores, las representaciones y los imaginarios sociales actuales. Es decir, como una base emocional para la reconstrucción de sí mismo en el presente a partir de una mirada crítica y reflexiva sobre el pasado que abre la posibilidad de construir un futuro (Boym, 2001). Con esta mirada, la nostalgia no es concebida solamente como un sentimiento, sino también como 
una práctica y un discurso sobre el mundo social y material presente que participa de la construcción identitaria de individuos y grupos (May, 2017).

Partiendo de esta idea, este artículo estudia las nostalgias expresadas como reveladores de espacios generacionales diferenciados en el barrio. Estos espacios generacionales son categorías elaboradas para el análisis. A partir de Mannheim (1928/1993), se aborda la generación como el resultado de una situación social $\multimap$ situación de generacióncompartida por un grupo de individuos que nacieron en una misma época histórica, generando un habitus de generación (Bourdieu, 1979). En este sentido, si bien la edad cuenta en el proceso de identificación a una generación, no se limita a este aspecto. Tener la misma edad no es suficiente para conformar una generación y distinguirla de otras. Una generación puede ser abordada como una construcción colectiva de un destino común en lo cual los individuos se reconocen y toman conciencia de perspectivas de vida compartidas.
Siguiendo esta línea, se discute la idea de nostalgia estructural propuesta por Herzfeld (1997) para referirse a su capacidad por reproducirse en cada generación sucesiva. Para el autor, existiría una transmisión intergeneracional de este sentimiento que se basaría en una retórica del cambio y de la decadencia compartida por las nuevas generaciones que sentiría un anhelo por un antes percibido como mejor. Sería un anhelo en particular por una reciprocidad entre las personas basada en el amor, el respeto o la honestad y que hoy, estaría desapareciendo. En su trabajo, Herzfeld asocia la nostalgia con la idea de permanencia. Este artículo se basa en el análisis de los datos empíricos contextualizados para discutir y complementar la idea de nostalgia estructural, mostrando que la nostalgia puede ser interpretada, pensada y experimentada de distintas formas según las personas y su rango etario.

\section{MÉTODO}

El artículo se basa en un trabajo de campo de dos años para identificar espacios generacionales estructurados por los sentidos dados a la nostalgia y a la experiencia del cambio. La estrategia metodológica se desarrolló entre 2019 y 2020 en dos momentos. Un primer momento (2019) consistió en la realización de talleres participativos sobre la temática "recordando mi barrio" destinados a adultos mayores de cada sector de estudio. Un segundo momento (2020) consistió en el desarrollo de un estudio intergeneracional dentro de familias de larga estancia de estos mismos tres sectores de estudio.

\section{Talleres: Recordando mi barrio}

Los talleres organizados en 2019 permitieron incorporar las opiniones de los participantes en la producción de conocimientos (Ward \& Barnes, 2016) y generar un lazo de confianza con los investigadores y entre los participantes, favoreciendo su participación y su empoderamiento en las actividades propuestas, donde una parte de los resultados sirvió para una exposición fotográfica sobre los recuerdos y las memorias de estos barrios. Los talleres fueron organizados gracias 
al apoyo logístico (i.e., difusión, invitación y prestación de sala) del Centro de Salud Familiar (CESFAM) de cada barrio.

La selección de los participantes se basó en tres requisitos: tener más de 65 años, vivir en el barrio/sector de estudio, y ser un habitante de larga estancia (más de 40 años). El taller se basó en cuatro actividades principales propuestas por un equipo de investigación interdisciplinar: (a) dibujar o escribir su barrio; (b) mapear los lugares pasados y presentes que los participantes consideran como estructurantes o importantes en su barrio; (c) llevar y narrar la historia de objetos personales (fotografías, objetos de lo cotidiano pasado o actual) vinculados con su experiencia del barrio; y (d) comentar fotografías actuales del barrio tomadas por el equipo de investigación para provocar reacciones emocionales y discusiones a propósito de su situación actual. Las sesiones fueron grabadas y posteriormente fueron transcritas por los miembros del equipo organizador. Además, para complementar los archivos orales, los miembros del equipo organizador tomaron notas de observaciones durante cada una de las actividades. Finalmente, el equipo tomó fotografías de las actividades. Las actividades se adaptaron a los diferentes grupos y contextos. En el barrio de la Esperanza, los talleres se desarrollaron en cuatro sesiones de dos horas con 13 participantes; en Barón, en seis sesiones de dos horas con diez participantes; y, en Playa Ancha Alto, hubo dos grupos diferentes: uno de ocho personas en seis sesiones de dos horas, y otro de 15 personas en dos sesiones de dos horas. Además, el equipo de investigación desarrolló entrevistas en profundidad para integrar a los habitantes que, por razones de salud, no pudieron participar en los talleres, o para profundizar en las experiencias de los participantes de los talleres anteriores. En estas instancias, además de preguntas orientadas hacia sus experiencias barriales pasadas y actuales, se propuso realizar de forma individual o en pareja, las mismas actividades que en el taller. Estas entrevistas fueron grabadas y transcritas. En total, se realizaron seis entrevistas en el barrio Esperanza, cuatro en Barón y tres en Playa Ancha Alto.

Un total de 51 personas participaron en esta primera etapa de investigación. La muestra es heterogénea en términos socioeconómicos, de edad (68 a 84 años), y en cuanto a las trayectorias vividas en cada barrio. Algunos nacieron en el mismo sector o incluso, a veces, en la misma casa donde aún viven. Otros llegaron de niños o adolescentes con sus padres. Finalmente, y como fue principalmente el caso de Playa Ancha Alto, algunos participantes llegaron a los nuevos edificios como adultos en espacios que no habitaban previamente. Durante las actividades propuestas durante los talleres y durante las entrevistas en profundidad, el equipo de investigación pudo recolectar distintos tipos de datos cualitativos. Desde informaciones sobre el barrio hasta sensaciones, emociones, y pensamientos de las personas participantes. Se pudo observar situaciones donde se entrelazan memorias individuales y colectivas, reminiscencias y rememoración, memorias y nostalgias. Estas actividades sirvieron de base para la realización posterior de exposiciones con las fotografías tomadas durante las sesiones y donde se recuperaron también algunas frases de los participantes sobre sus recuerdos del barrio de antes y hoy. 


\section{Estudio intergeneracional}

Un segundo momento se desarrolló en 2020 y consistió en un estudio intergeneracional con familias de los tres sectores de estudio. Este estudio se centró en las experiencias nostálgicas del cambio socio-urbano expresadas por miembros de distintas generaciones de estas familias. La selección de las familias se realizó gracias a la ayuda de las personas que participaron en los talleres en 2019 y a los CESFAM de los sectores de estudio. Las familias que participaron de este estudio eran familias de larga estancia (más de 40 años) compuestas de al menos dos generaciones de personas mayores de 18 años, en las cuales algunos de sus miembros han vivido a proximidad en la misma casa, en el sector estudiado que pueden pertenecer a la familia directa o indirecta.

Se realizaron entrevistas en profundidad con 2 a 5 miembros de las mismas familias perteneciendo a generaciones distintas. En el contexto complejo de la pandemia asociada con el COVID19, las entrevistas fueron desarrolladas en línea a través de una plataforma virtual o una aplicación de redes sociales. En la mayoría de los casos, conocíamos previamente al menos un miembro de la familia gracias a los talleres, lo que facilitó el acceso y el buen desarrollo de las entrevistas. Las entrevistas fueron grabadas y transcritas. Estas tomaron la forma de un relato de vida donde se abordó los recuerdos individuales, familiares y colectivos vinculados con el barrio, así como la percepción que el sujeto tiene del barrio hoy. Según la situación, las entrevistas fueron individuales o grupales (e.g., el padre y la madre, o el padre o madre y su hija/o). Estas distintas configuraciones permitieron identificar y analizar los modos de transmisión de la nostalgia y la manera de interpretar esta transmisión por cada una de las generaciones y de las personas, visibilizando acuerdos o desacuerdos en los sentidos y significados otorgados a los recuerdos. En el cerro Esperanza, participaron dos familias compuestas por tres generaciones distintas. En el cerro Barón, participaron dos familias: una compuesta por dos generaciones y otra por tres generaciones. Finalmente, en el sector Playa Ancha Alto, participaron tres familias: dos compuestas por tres generaciones y una por dos generaciones. En total se realizaron entrevistas a 20 personas de generaciones distintas y con perfiles sociales y trayectorias familiares diferentes.

Esta segunda estrategia metodológica implementada en los tres barrios permitió acceder a repertorios memoriales y afectivos tanto individuales como familiares, algunos compartidos y otros en conflicto. A partir de este trabajo, se identificó progresivamente actitudes y reacciones distintas según las generaciones de pertenencia frente a la evocación de pasados, así como también, se reconocieron formas de relacionarse con el barrio y sus cambios.

\section{Consideraciones éticas}

Las personas participaron de forma voluntaria y firmaron un consentimiento informado antes el inicio de cada sesión de taller o de cada entrevista. En el marco del taller, los participantes firmaron además un consentimiento por el uso de la grabación y de las fotografías en el marco de la elaboración de la exposición de 
pósteres fotográficos final. En el marco de las medidas COVID19, los participantes del estudio intergeneracional expresaron de forma oral su acuerdo. Este acuerdo oral fue grabado. Todos los datos producidos en esta investigación fueron tratados de forma anónima, privada, y absolutamente confidencial. En este trabajo, todos los nombres han sido cambiados para asegurar el anonimato de los participantes.

\section{RESULTADOS}

El artículo se basa en los resultados de esta doble estrategia metodológica para explorar los espacios generacionales barriales como espacios sociales en los cuales se comparte comportamientos, sentimientos y actitudes. A partir del cruzamiento de los datos de los talleres y las entrevistas asociadas (2019) y del estudio intergeneracional (2020), el artículo identifica tres espacios generacionales: (a) un espacio que se denominó como "primeras generaciones", compuesto por personas adultas mayores de 60 años que comparten una nostalgia del barrio de antes y que expresan muchas veces una visión distópica de su barrio actual en comparación de un pasado que juzgan mejor; (b) el espacio de las "segundas generaciones" compuesto por personas entre 35 y 60 años y que expresan una nostalgia por el barrio relacionado con su juventud y; (c) el espacio de las "nuevas generaciones", está compuesto por los nietos de las primeras generaciones que tienen entre 20 y 35 años y se caracterizan por apropiarse y reinterpretar las nostalgias heredadas de las otras generaciones en relación con sus propias experiencias. Estos tres tipos de espacios generacionales son analizados a continuación.

\section{Primeras generaciones: El lamento por la pérdida}

Una mayoría de las personas mayores de 60 años que participaron en el estudio expresan una nostalgia por el barrio de antes (8 participantes en los talleres y 2 adultos mayores participando en estudio intergeneracional no expresaron ninguna nostalgia), basada en una comparación entre un tiempo pasado y el presente. Sus narrativas revelan una añoranza por los valores y los estilos de vida pasados compartidos por una generación de personas que hoy está desapareciendo. Se revela por ejemplo cuando los participantes del cerro Esperanza explican que, a pesar de vivir con menos recursos económicos, antes conocían una vida social mucho más solidaria. Se observa cuando los participantes del cerro Barón se refieren a lo que llamaban "la modernización" de su barrio y que tiene que ver con la proliferación reciente de edificios residenciales en altura. Emerge finalmente también en los recuerdos de los habitantes de Playa Ancha Alto, la idea de un barrio que era antes mucho más convival, familiar y seguro, con una convivencia sana. 
Cuando describen el barrio, los adultos mayores del estudio se refieren a sus habitantes, su familia, y sus vecinos. Su nostalgia por el barrio se basa en una añoranza por el barrio como espacio relacional desaparecido. Es decir, un espacio social compuesto por personas y familias que hoy han desaparecido o se han ido del barrio. En este contexto, expresan un malestar frente a los cambios en las reglas de convivencias que no quieren o no pueden compartir con las generaciones ulteriores. Esta situación se refleja en los relatos por ejemplo de Yesika, 73 años, habitante del cerro Barón. Nativa del cerro, vive hoy en la casa familiar donde creció. Comparte su casa con su hermana, y con su hija de 35 años y su pareja. Conoce muy bien su barrio y las familias antiguas del sector. Durante la entrevista, expresó una fuerte añoranza por la vida social pasada:

antes era más familiar. [...] Ahora del punto de vista de cómo lo ve [mi hija] es totalmente diferente porque ella como es más jovencita no vio esa parte donde todas las familias eran unidas. Eran muy poquitas, pero eran unidas. Todos nos llamábamos por los apellidos, tal familia con tal nombre. No eran así individuales los niños [...]. [Hoy] no es como antes. Hay una gran diferencia, entre lo que éramos antes nosotros y lo que son ahora los jóvenes. (Yesika, 73 años, habitante del cerro Barón).

En el relato de Yesika, la nostalgia aparece desde la comparación entre sus experiencias de infancia y lo que observa de la vida de los niños hoy. En su narrativa, se genera así un quiebre generacional insuperable entre un nosotros compuesto por las personas de su generación y otros que son las otras generaciones. Sin embargo, este quiebre nace también de la desaparición de su propia generación, y más precisamente las personas antes cercanas. En el caso de Yesika, se trata de su madre. Esta añoranza por su familia se expresó en particular cuando compartió recuerdos de momentos en su casa:

Venía mucha gente a esta casa, mucho, mucho, por eso es el recuerdo de eso que siempre había algo, muchas frutas que se regalaba. Mi mamá las repartía para las distintas familias, entonces hay muchos recuerdos. Yo, la verdad, es que no creo que dejaría esta casa. [...]Es una casa con recuerdos que no se puede dejar. (Yesika, 73 años, habitante del cerro Barón). La nostalgia por la vida social barrial se basa en experiencias familiares propias que hace que estas personas, si bien critican la situación presente, se sienten pertenecer al barrio desde sus recuerdos e historias. Una situación similar está descrita por Katrina, 62 años, viviendo en Playa Ancha Alto desde los años 1972 con sus padres y 16 hermanos. Hoy, sigue viviendo en la casa familiar con algunos de sus hermanos, su madre de 100 años y su hijo de 29 años. Si bien es más joven que los otros participantes de estas primeras generaciones, la asociamos a este grupo debido a su relación con su nostalgia y sus experiencias de los cambios barriales. Como Yesika, expresa durante la entrevista una fuerte nostalgia por la vida social barrial pasada:

Quienes estábamos, varias que vivíamos acá y también hay gente que se ha ido. Ahí nos juntábamos en la escala. La gente que vivía donde mi amiga la vecina, había gente que vivía porque ella arrendaba y también se fueron. Así que quedamos pocos (Katrina, 62 años, habitante de Playa Ancha Alto). 
En este marco, su nostalgia no se vincula solamente con su infancia, sino también con la pérdida de su marido, de su hermana y de hermanos. Vinculados con el barrio, estas pérdidas generaron un quiebre en las relaciones vecinales que habían desarrollado:

De ahí cuando falleció mi esposo y después mi hermana, ya como que no nos juntamos mucho. [...] Cuando falleció mi esposo ya no nos acercábamos a la escala. [...] Mis hermanas fallecieron también, dos hermanas con las que yo compartía, nos llevábamos por un año. Y aquí ya no, o sea igual el barrio no es lo mismo, no, no (Katrina, 62 años, habitante de Playa Ancha Alto).

Los dos ejemplos hacen aparecer un espacio generacional basado en una nostalgia por una vida social desaparecida debido a la pérdida de sus principales actores. Sin embargo, si bien sus añoranzas se refieren de forma permanente a su generación de habitantes, diferenciándose de las otras generaciones que vinieron después, se fundan principalmente en el sentimiento de pérdida asociado con su entorno social más cercano que, para estas personas, conformaba lo que definen como "su barrio". En este marco, los distintos relatos recogidos durante el trabajo de terreno concuerdan en una misma nostalgia por el barrio de antes, como espacio relacional desaparecido. Pero este sentimiento de pérdida no se sustenta en las mismas experiencias pasadas ni los mismos entornos sociales. Encontramos una situación similar en el caso de las segundas generaciones que están conformadas por una nostalgia asociada con la pérdida de la juventud.

\section{Segundas generaciones: Lamento por la juventud}

Si bien las segundas generaciones, generalmente hijas e hijos de las personas que conforman las primeras generaciones, expresan también una nostalgia por el barrio de antes, esta emoción no se refiere ni a los mismos recuerdos ni a las mismas personas. Este espacio generacional se configura principalmente desde un lamento por una juventud perdida y que, para estas personas, era central en la vida barrial que tenían. Se puede referir tanto a su propia juventud como a la juventud de sus hijas e hijos hoy adolescentes o adultos. En ambos casos, a la diferencia del primer grupo, la nostalgia no se refiere a personas desaparecidas, sino a la irreversibilidad del tiempo que pasa y que se refleja en las personas que siguen componiendo el espacio social barrial. Por otra parte, como sus padres, asocian con el barrio de antes ideas y valores que hoy estarían desapareciendo tales como la confianza, la seguridad, la solidaridad. Sin embargo, asocian con estas ideas y estos valores recuerdos y experiencias de infancia o de la infancia de sus hijas e hijos.

Para ilustrar esta forma nostálgica de relacionarse con el barrio, se puede dar el ejemplo de Francisco, 50 años, que nació en el cerro Esperanza y que, luego haber vivido un tiempo en otra ciudad, vive hoy en el barrio, en la casa familiar junto con su madre y algunos de sus hermanos. Describe su barrio como un espacio familiar compuesto por personas "nacidas y criadas" de este sector y en mayoría adultas mayores. En su relato, retoma las críticas hechas por las personas de las primeras generaciones acerca de la pérdida de las relaciones sociales de antes. Sin embargo, juzga que no se ha perdido totalmente: 
las personas que todavía viven acá en el barrio, gente que igual mantiene su amabilidad, ese espíritu social, que bueno si uno se encuentra en algún momento, uno tiende a conversar con los vecinos. Todavía no se pierde la comunicación a pesar de que ya no nos vemos como antes (Francisco, 50 años, habitante del cerro Esperanza). Para Francisco, el problema actual del barrio tiene que ver con el envejecimiento de sus habitantes que conduce a una pérdida progresiva de la vida barrial como lo había conocido antes. Mientras las primeras generaciones sienten nostalgia por la pérdida, las personas que pertenecen a las segundas generaciones experimentan esta emoción desde la percepción del envejecimiento. Esta preocupación se observa en este relato:

este se hizo un barrio viejo entre comillas porque hay mucha gente adulta [mayor]. Entonces la gente adulta está más ligada a las enfermedades. Ya no se trasladan como antes y ya no se ve tanta juventud ¿ya? No se ven tantos niños, entonces no hay como tanto ambiente de calle. La gente está más en la casa, más que nada y bueno que además los niños como se sabe están más ligados a su celular, a los juegos y eso los mantiene más adentro de sus casas, entonces eso es lo que igual se echa de menos. (Francisco, 50 años, habitante del cerro Esperanza).

Frente esta situación, su nostalgia por el barrio de antes se activa cuando recuerda su infancia y la vida social que tenía durante este periodo:

es un muy buen barrio tranquilo, donde la mayoría de las familias se conoce, donde todas las generaciones se reunían en torno a la calle, a todas las actividades desde niños. Los adultos igual de cierta forma se juntaban, se hicieron muchas amistades de todas las generaciones y además que nosotros tenemos un patio que es un estadio. Antiguamente era un estadio, el estadio ferroviario, y tenía acceso libre, donde nosotros podíamos contar con jugar con los árboles, en los cerros, escalar, em... Teníamos dos canchas, una cancha de basquetbol y una cancha de futbol. Ese era nuestro patio que teníamos en el fondo, con toda la tranquilidad, con toda la libertad ¿ya? Porque desde niños pasábamos ahí y la mayor parte del tiempo y bueno, en diferentes sectores, además que tenemos un club deportivo donde se hacían campeonatos todos los veranos. (Francisco, 50 años, habitante del cerro Esperanza).

Una libertad, una felicidad y un entretenimiento que, según Francisco, hoy no son más posible en gran parte por el envejecimiento de sus amigos de su edad y de sus padres. Sin embargo, esta nostalgia no significa necesariamente una falta de compromiso con el presente y abandono de todo anhelo por el futuro. En efecto, antes de la pandemia, Francisco participó activamente en la organización de vecinos para la seguridad del barrio. En el contexto de la pandemia, esta organización sigue funcionando a través de las redes sociales y permite a los habitantes quedar en contacto. En suma, la nostalgia por un barrio más solidario, como era en su infancia, lo ha conducido a actuar para reencontrar este espacio social que estaba desapareciendo.

Rosa, 55 años, habitante del sector Playa Ancha Alto, comparte un relato nostálgico similar orientado hacia su infancia. Llegó en este barrio en 1976 con sus padres y sus hermanos. Hoy vive en una casa con su marido y sus dos hijos, ubicada cerca del 
departamento de sus padres. Comparte la mirada crítica de las primeras generaciones acerca de la falta de relación vecinal y de solidaridad hoy, en comparación del pasado. Sin embargo, al contrario de sus padres o de otras personas de las primeras generaciones, la nostalgia que expresa se refiere ante todo a su infancia, antes de llegar en el barrio:

Es la gran diferencia de los barrios que vivíamos antes, porque yo recuerdo de que el barrio que vivíamos cuando éramos chicos, que todo el mundo saludaba a todo el mundo, todo el mundo se conocía, para el año nuevo era una cosa de celebrar todas las familias celebraban con las familias de al lado, nos saludábamos todos, nos abrazábamos todos, eso cambio yo creo hace ya 30 años más o menos que nosotros llegamos el 76 acá y ya no se dio la misma empatía el mismo cariño por el vecino como existía antes (Rosa, 55 años, habitante de Playa Ancha Alto).

Rosa comparte así la nostalgia por la pérdida de los valores relacionados con la reciprocidad, la solidaridad y la seguridad, lo que a su parecer ha conocido durante su infancia y que los niños hoy no pueden vivir:

la infancia, la niñez nuestra fue hermosa, con seis hermanos, con un papá y una mamá siempre presente. Tratamos de que prevalezca eso, nosotros en la vida que estamos teniendo ahora con nuestros hijos, con nuestros nietos. La diferencia es que ahora no podemos dejarlos solos en la calle o que salgan a jugar a una plaza como se hacía antes. Antes, ellos estaban seguros de que los hijos salían a jugar o los niños salían a jugar a la pelota, las mujeres a juntarse con las amigas. Eso ahora no se ve dentro de la niñez de este barrio (Rosa, 55 años, habitante de Playa Ancha Alto).
Como Francisco, la nostalgia expresada por Rosa no ha significado un deseo de regreso al pasado sino más bien una adaptación al presente y una proyección hacia un futuro posible. Valorizando el rol de sus padres y de su familia en la realización de su infancia feliz, Rosa busca transmitir la importancia de la unidad y de la solidaridad familiar a sus hijos. En este espacio generacional, la nostalgia por el barrio se basa en los mismos valores y las mismas ideas heredadas de sus padres. Sin embargo, no se relaciona con las mismas experiencias ni los mismos recuerdos. Se trata de una nostalgia mucho más orientada hacia una vida familiar cambiada que hacia un ideal de vida barrial desaparecido (como en el caso de las primeras generaciones). Por otra parte, en los relatos, la nostalgia expresada toma una dimensión más reflexiva, conduciendo a actuar para adaptarse a los cambios generados por el envejecimiento.

\section{Las nuevas generaciones: La nostalgia reinterpretada}

El espacio de las nuevas generaciones se basa en una apropiación y una reinterpretación de la nostalgia por el barrio de las primeras y segundas generaciones. En este sentido, presenta una diversidad más grande de formas de pensar y relacionarse con el barrio. A partir del estudio intergeneracional realizado en 2020, el análisis conduce a la identificación de dos tipos principales de reinterpretación de la nostalgia heredada de las otras generaciones: (a) por un lado, la expresión de una nostalgia basada en imaginarios desarrollados a partir de los recuerdos de las otras generaciones y; (b) por otro lado, la expresión de una anti-nostalgia frente a la nostalgia compartida por las otras generaciones. 
El caso de Josefa, 23 años, habitante del sector Playa Ancha Alto, permite ilustrar el primer tipo de reinterpretación de la nostalgia heredada por las otras generaciones de su familia. Josefa es estudiante y vive con sus padres y su hermano menor. Gran parte de su familia vive en la misma calle y su abuela materna vive en el barrio desde 1964. Josefa vivió en España de sus siete a sus quince años. Hoy expresa una fuerte nostalgia por la vida familiar que ha vivido a través de los recuerdos y de las historias de sus padres. Intenta reproducir y reencontrar este tiempo perdido con su familia en Chile durante su periodo de vida en España. Se trata por lo tanto de una nostalgia basada en imaginarios por momentos que no ha conocido directamente. Momentos y vida familiar que vive a través de los relatos de sus padres, tíos, y tías. Su relato muestra que estando en España quería volver en su barrio para reencontrarse con el resto de su familia:

quería saber de dónde venía porque como me fui tan chica como que no conozco de dónde vengo. A mi familia no la veo hace mucho tiempo, entonces volver sí que fue como una sensación de sí, yo pertenezco como a este lugar, como que este es mi hogar, aquí está mi familia. Me sentía bien porque era lo que yo quería hacer en ese momento y yo me vine con esa visión de volver a mi hogar. (Josefa, 23 años, habitante de Playa Ancha Alto).

Este deseo de regreso se basa en los recuerdos de la vida familiar que tenía antes de los siete años, pero también de las historias relatadas por sus padres: cuando [mis padres] se juntaban, siempre se hablaba de Chile. En la familia igual, yo tenía recuerdos muy puntuales como a lo mejor de las vistas. Me acordaba de cómo era la entrada de mi sector, que era una bajada que hay un mural grande. Me acordaba de la plaza, de la casa de mis abuelos. Cosas muy puntuales o a veces fotos. Teníamos, tenemos un montón de fotos y las fotos también me ayudaban así "oh sí, me acuerdo de este lugar" "oh, sí me acuerdo de esta persona". Pero, tampoco son tanto recuerdos, sino es más lo que me van como contando, lo que me fueron contando en ese momento (Josefa, 23 años, habitante de Playa Ancha Alto).

Su nostalgia por un tiempo no conocido se vuelve más presente cuando se encuentra con sus primos de su edad y no puede compartir los mismos recuerdos ni comportamientos en el barrio:

ellos sí, vivieron la infancia acá, conocen a la gente, conocen al resto de los jóvenes. [...] Me conocen de pequeña, pero después, desaparecí un montón de tiempo, pero volví. Entonces, a lo mejor mi cara, no es lo mismo, entonces yo no puedo ir tan libremente (Josefa, 23 años, habitante de Playa Ancha Alto).

Frente esta situación, su nostalgia le permite proyectarse en el futuro desde un pasado imaginado y deseado. En este sentido, su nostalgia le sirve hoy de recurso para construirse como persona en relación con su familia y su barrio.

Otra reinterpretación de la nostalgia de las otras generaciones está ilustrada por el caso de Iván, 29 años y habitante de Playa Ancha Alto. Originario del barrio, vivió un periodo en otro sector de la ciudad, y volvió a vivir en la casa de sus padres en 2017, luego de la separación con su pareja. Luego de esta ruptura, Iván se reencontró con su barrio y está reconstruyendo su sentido de pertenencia 
desde sus acciones y su compromiso con los vecinos, participando activamente en la vida social y política del barrio, desarrollando proyectos comunitarios en conjunto con las instituciones locales y los habitantes. En este marco afirma que su trabajo comunitario no se basa en un deseo de regreso a estilos de vida pasados, como a su parecer le gustaría las otras generaciones:

Es como súper cliché decir que todo tiempo pasado fue mejor, pero eso es un poquito lo que tratamos de revertir. [...] Mi mamá está muy arraigada en eso: "no, pero es que nosotros salíamos a la calle, ahora no se puede y antes nosotros". Pero, yo le digo "¿por qué no lo hacemos?" O sea, ahora pensamos hacía el futuro y lo logramos de hecho (Iván, 29 años, habitante de Playa Ancha Alto).
En este marco, Iván expresa claramente una forma de anti-nostalgia: el sentido que da a sus acciones en el barrio se arraiga en el presente y no se orientan hacia un pasado deseado.

ahora tengo la posibilidad de identificarme con un barrio en el que yo viví y que hoy también, mi hijo, por ejemplo, los hijos de mis amigos están viviendo, están haciendo barrio. Entonces, de la nostalgia pasamos ya al seguir hacía adelante, como a ver que se nos viene (Iván, 29 años, habitante de Playa Ancha Alto).

Este rechazo de la nostalgia se transforma en recurso para actuar en el barrio. Frente a las nostalgias de las primeras y segundas generaciones, propone pensar en y desde el presente y hacia el futuro. Se sirve de estas nostalgias para proponer soluciones $\mathrm{y}$ futuros posibles.

\section{CONCLUSIONES}

A partir de un trabajo de campo con habitantes y familias de larga estancia en tres barrios de Valparaíso, el artículo analizó los fundamentos de las nostalgias expresadas por el barrio de antes, las dimensiones estructurales de esta nostalgia, los sentidos, y los significados que personas de distintas generaciones asocian con este sentimiento. Los resultados muestran que, si bien la nostalgia por el barrio de antes es globalmente compartida, no toma la misma forma ni se refiere a las mismas realidades dependiendo de los habitantes. De una generación a otra, este sentimiento es progresivamente reinterpretado según las propias experiencias pasadas de las personas. Así, la nostalgia no significa solamente un deseo de regreso, sino también una cierta reflexividad en relación con el presente y el futuro (Boym, 2001; Pickering \& Keightley, 2006). El lamento de las primeras generaciones revela una mirada crítica del presente en comparación a un pasado deseado. Sin embargo, este deseo no significa una búsqueda de regreso al idéntico, sino más bien un recurso para pensarse y vivir en relación con el presente. El lamento de las segundas generaciones también muestra una mirada reflexiva sobre el tiempo, el envejecimiento, y los cambios sociales y familiares que han vivido en el barrio. Para ellos, hablar de nostalgia por el barrio significa pensar sus propios cambios. Finalmente, las nuevas generaciones toman distancia y reinterpretan las nostalgias 
de las generaciones anteriores según sus propias experiencias y expectativas. En suma, si bien la nostalgia por el barrio puede ser identificada como una nostalgia estructural, en el sentido que se transmite intergeneracionalmente, toma distintas formas.

Por otro lado, estudiar las nostalgias por el barrio según las generaciones de pertenencia permite abordar los espacios vividos y percibidos de estos habitantes (Lefebvre, 1974), mostrando las distintas formas de pensar y vivir el barrio y sus cambios. Hace así aparecer un quiebre generacional a escala barrial basado en estas distintas formas de entender la nostalgia. En este marco, cada espacio generacional tiene su forma de experimentar e interpretar los cambios. Las primeras generaciones se centran en la pérdida de los estilos de vida de antes y de las personas que, según ellas, hacían barrio. Las segundas generaciones asocian la idea de barrio como la familia, y más específicamente, sus experiencias durante la infancia. Finalmente, las nuevas generaciones buscan dar un nuevo sentido al barrio a partir de los recuerdos y las historias transmitidos por las generaciones anteriores. En este marco, esta proyección hacia otro futuro buscado por las nuevas generaciones puede entrar en contradicción con la forma en que las otras generaciones piensan su pasado, presente y futuro. En suma, estudiar las nostalgias permite comprender las tensiones y desacuerdos existentes entre personas de distintas generaciones a escala barrial y también a escala intrafamiliar. En este sentido, las nostalgias por el barrio dicen de los problemas vividos por estos habitantes acerca de las relaciones y tensiones intergeneracionales dentro de un mismo territorio. La nostalgia muestra que se superponen espacios sociales, generacionales y emocionales diversos que muchas veces no se entienden ni interactúan. Este artículo buscó mostrar que para favorecer diálogos intergeneracionales es necesaria la comprensión de las emociones expresadas por las personas que componen estos espacios. Esto demuestra la importancia de analizar las emociones y los múltiples sentidos que las personas asocian para comprender estos fenómenos sociales. $\mathrm{Si}$ bien los resultados de esta investigación se fundan en una muestra socioeconómica heterogénea y enmarcada en contextos socio-territoriales distintos, sería interesante poder complementar este trabajo con datos empíricos enmarcados en otros contextos socio-urbanos y con habitantes con otras trayectorias socio-residenciales, permitiendo a su vez una comparación de los resultados y una generalización más extensa. Pese a esto, los resultados presentados en este artículo aportan elementos de comprensión no solamente de esta nostalgia, sino también de los espacios vividos y percibidos de la ciudad contemporánea (Lefebvre, 1974), comprensión que no se podría lograr sin tomar en cuenta estas emocionalidades. 


\section{REFERENCIAS}

Aguilar, M. A., \& Soto, P. (Eds). (2013). Cuerpos, espacios y emociones: Aproximaciones desde las ciencias sociales. M-A Porrúa.

Appadurai, A. (1996). Modernity at large. Cultural dimensions of globalizations. University of Minnesota Press.

Bauman, Z. (2017). Retrotopía. Paidós.

Bourdieu, P. (1979). La Distinction. Critique sociale du jugement. Edition de Minuit.

Blunt, A. (2003). Collective memory and productive nostalgia: Anglo-Indian homemaking at McCluskieganj. Environment and Planning D: Society and Place, 21(6), 717-738. https://doi.org/10.1068/d327

Bonnett, A., \& Alexander, C. (2013). Mobile nostalgias: Connecting visions of the urban past, present and future amongst ex-residents. Transactions of the Institute of British Geographers, 3\&(3), 391-402. https://doi.org/10.1111/j.1475-5661.2012.00531.x

Bonnett, A. (2015). The geography of nostalgia. Taylor \& Francis.

Boym, S. (2001). The future of nostalgia. Basic Books. Campos, L., Silva, R., \& Gaete, M. (2017). El rol de las emociones y los afectos en la producción del hábitat y el territorio. Revista INVI, 32(91), 9-21. https://doi.org/10.4067/S071883582017000300009

Colin, C. (2017). La nostalgia en la producción urbana: La defensa de barrios en Santiago de Chile. Revista INVI, 32(91), 91-111. http://doi.org/10.4067/S0718-83582017000300091
Colin, C., Gervais-Lambony, P., Hirai, S., \& Pinto, C. (2019). Les nostalgies dans la ville contemporaine: Pistes de recherche. Cybergeo: European Journal of Geography, Artículo 904.

https://doi.org/10.4000/cybergeo.32469

Davis, F. (1979). Yearning for yesterday. A sociology of nostalgia. Free Press.

Herzfeld, M. (1997). Cultural Intimacy:Social poetics in nation-state. Routledge.

Hochschild, A. R. (1983). The managed heart. Commercialization of human feeling. University of California Press.

Kemper, T. (1978). A social interactional theory of emotions. John Wiley \& Sons.

Kitson, J., \& McHugh, K. (2015). Historic enchantments-materializing nostalgia. Cultural Geographies, 22(3), 487-508. https://doi.org/ $10.1177 / 1474474014549946$

Lefebvre, H. (1974). La production del'espace. Anthropos. Letelier, F. (2018). El barrio en cuestión. Fragmentación y despolitización de lo vecinal en la era neoliberal. Scripta Nova, 23(602). https://doi.org/10.1344/sn2018.22.21518

Lutz C., \& White G. (1986). The anthropology of emotion. Annual Review of Anthropology, 15, 405-436. https://doi.org/10.1146/annurev.an. 15.100186 .002201

Mannheim, K. (1993). El problema de las generaciones. Revista Española de Investigaciones Sociológicas, (62), 193-242.

http://www.reis.cis.es/REIS/PDF/REIS_062 _12.PDF (Trabajo original publicado en 1928). 
Massey, D. (2005). For space. Sage.

May, V. (2017). Belonging from afar: Nostalgia, time, and memory. The Sociological Review, 65(2), 401-415. https://doi.org/10.1111/1467954X.12402

Pickering, M., \& Keightley, E. (2006). The modalities of nostalgia. Current Sociology, 54(46), 919-941. https://doi.org/10.1177/ 0011392106068458

Pincheira, I. (Ed.). (2019). Emociones en Chile contemporáneo. Metales Pesados.

Ramírez, P., \& Aguilar, M. (Eds.). (2006). Pensar y habitar la ciudad: Afectividad, memoria y significado en el espacio urbano contemporáneo. Anthropos; UAM Iztapalapa.

Scribano, A., \& Aranguren, M. (Eds.). (2017). Aportes a una sociología de los cuerpos y las emociones desde el Sur. Estudios Sociológicos.
Shaw, C., \& Chase, M. (Eds). (1989). The imagined past: History and nostalgia. Manchester University Press.

Tapia, V. (2018). Geografía de la contención: El rol de las políticas de escala barrial en el Chile neoliberal. Scripta Nova, 22(592). https://doi.org/10.1344/sn2018.22.20272

Thoits, P. (1989). The sociology of emotions. Annual Review of Sociology, 15, 317-342. https://doi.org/10.1146/annurev.so.15.08018 9.001533

Ward, L., \& Barnes, M. (2016). Transforming practice with older people through an ethic of care. British Journal of Social Work, 46(4), 906-922. https://doi.org/10.1093/bjsw/bcv029

Manuscrito recibido: 26-02-2021

Manuscrito aceptado: 12-07-2021 